\title{
Silicon-Based Quantum Mechanical Tunnel Junction for Plasmon Excitation from Low-Energy Electron Tunneling
}

\author{
Fangwei Wang, ${ }^{\#}$ Yan Liu, ${ }^{\#}$ Thorin Jake Duffin, ${ }^{\#}$ Vijith Kalathingal, Siping Gao, Wenrui Hu, \\ Yongxin Guo, Soo-Jin Chua, and Christian A. Nijhuis*
}

Cite This: ACS Photonics 2021, 8, 1951-1960

Read Online

ACCESS | 岁 Metrics \& More | 回 Article Recommendations

Supporting Information

ABSTRACT: Light emission from metal-insulator-semiconductor junctions (MISJs) has been explored for decades as a possible on-chip light source; however it is not clear whether the mechanism of light emission is plasmonic in nature or is dominated by electroluminescence. Previous studies only investigated silicon with low doping levels, but here we show that only highly doped silicon allows us to excite surface plasmon polaritons (SPPs) in MISJs via inelastic tunneling. This paper describes the mechanism of charge transport and light emission from siliconbased $\mathrm{Au}-\mathrm{SiO}_{2}-\mathrm{nSi}$ MISJs as a function of the doping level $N_{\mathrm{d}}$ varying from $1.6 \times 10^{15} \mathrm{~cm}^{-3}$ to $1.0 \times 10^{20} \mathrm{~cm}^{-3}$. At low doping

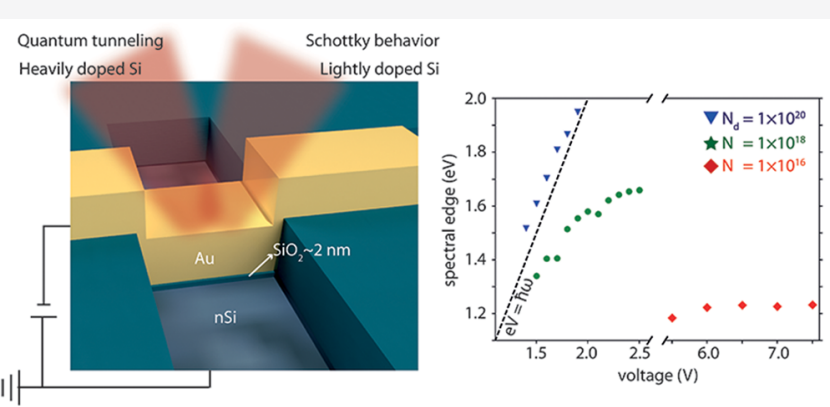
levels $\left(N_{\mathrm{d}} \sim 10^{15} \mathrm{~cm}^{-3}\right)$, the MISJs behave as Schottky diodes, and the mechanism of light emission involves a radiative recombination of electrons and holes from minority carrier injection under high applied bias $(>5.5 \mathrm{~V})$. With increasing doping levels, the current-voltage characteristics of the MISJs change, resulting in symmetrical current-voltage curves with parabolic conductance behavior characteristic of quantum mechanical tunneling. MISJs with the highest doping level $\left(N_{\mathrm{d}} \sim 10^{20} \mathrm{~cm}^{-3}\right)$ are dominated by quantum mechanical tunneling, and light emission originates from radiative decay of surface plasmon polaritons (SPPs) via scattering at threshold voltages as low as $1.5 \mathrm{~V}$. Our simulations indicate that tunneling over the thin $\mathrm{SiO}_{2}$ barrier between the Au and highly doped $\mathrm{nSi}$ excites a hybrid-SPP mode localized to the Au whose dispersion depends on the effective index induced by the $\mathrm{SiO}_{2}-\mathrm{nSi}$ interface. Our studies show that $\mathrm{Si}$ needs to be sufficiently doped to be conductive enough to enable SPP excitation via inelastic tunneling.

KEYWORDS: tunnel junction, plasmon excitation, hybrid plasmon modes, metal-insulator-semiconductor junction, carrier concentration

E lectrical excitation of surface plasmon polaritons (SPPs) has the potential for integration into technologies that shrink down the dimensions of optoelectronic circuity, with applications in sensing and interfacing technologies. ${ }^{1-3}$ In this context, metal-insulator-metal tunneling junctions (MIMTJs) are interesting to study as they, under an applied bias, can excite SPPs via inelastic tunneling with efficiencies exceeding $1 \%{ }^{4-7}$ These MIM-TJs are composed of two metal electrodes, which limits the ease of integration into complementary metal oxide semiconductor (CMOS) technology $y^{7-10}$ as well as their application by virtue of the inherently lossy plasmonic response of metals. ${ }^{11,12}$ Therefore, replacing one or both of the electrodes with $\mathrm{Si}$ or CMOS compatible materials would pave the way toward CMOS compatible electrically driven SPP sources. ${ }^{13-15}$ Here we report SPP excitation and light emission from silicon-based hybrid plasmonic metal-insulator-semiconductor junctions (MISJs) with low drive voltages. Only MISJs with a highly doped Si electrode can excite SPPs excited via inelastic tunneling with low drive voltages $(1.5 \mathrm{~V})$, while electroluminescence (electron-hole recombination) from minority carrier injection is the main mechanism of light emission from junctions with low doping levels (i.e. Schottky diodes), requiring high drive voltages $(>5.5 \mathrm{~V})^{16}$ to overcome the Schottky barrier and to obtain large enough electron-hole recombination rates to observe light emission. To quantitively demonstrate the quantum mechanical tunneling excitation of the hybrid-SPP modes in highly doped MISJs, we analytically calculated the local density of optical states (LDOS) of the MISJs and corroborated the electromagnetic response of the MISJ using finite element method (FEM) analysis. This work gives new insights into light-matter interactions in Si-based plasmonic tunnel junctions, which helps to guide future efforts toward the development of CMOS compatible plasmonicelectronic circuitry.

Received: December 16, 2020

Published: June 9, 2021 
Light emission from MISJs has been studied before, ${ }^{17-36}$ but so far all MISJ studies have used Si with low doping levels $\left(N_{\mathrm{d}}\right.$ $=10^{14}-10^{16} \mathrm{~cm}^{-3}$ ) and only measurable amounts of light could be detected by applying large voltages (3-11 V). ${ }^{17-32,34-37}$ Although the majority of these studies claim electroluminescence from electron-hole recombination as the origin of light emission, ${ }^{17-23,28-32,34-40}$ others ${ }^{25,2741,42}$ disagree and claim that light emission occurs via radiative decay of SPPs excited via inelastic tunneling. It is well-known that MISJs with low doping levels result in Schottky diodes (Figure 1a) where the most likely mechanism for light emission
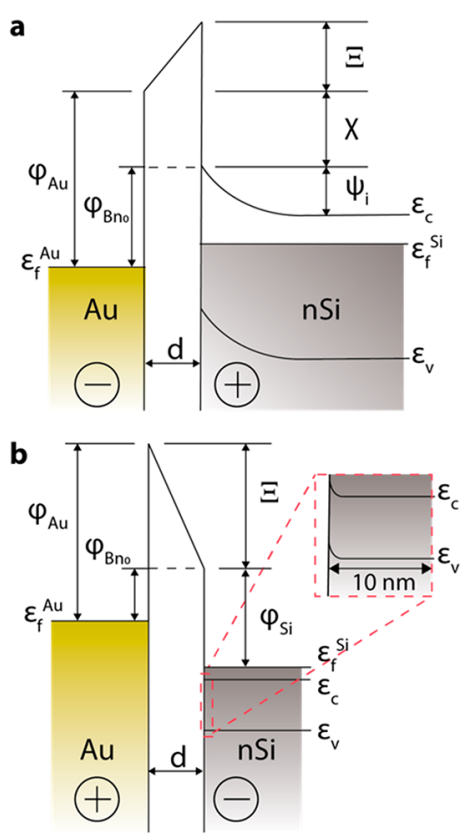

Figure 1. (a) Energy level diagrams for an MISJ with a Schottky interface and (b) for an MISJ dominated by quantum tunneling. In all of our experiments, the Si electrode was connected to the ground and the $\mathrm{Au}$ electrode was biased.

involves electroluminescence from electron-hole recombination, as well as light emission from dielectric breakdown, for applied bias voltages of $>2 \mathrm{~V}^{43}$ Moreover, it is unlikely that tunneling barriers (which typically consist of $1-3 \mathrm{~nm}$ thick insulators) can withstand such large voltages given that $\mathrm{SiO}_{2}$ has a typical electrical break down strength on the order of 4 $\mathrm{GV} / \mathrm{m} .^{44}$ In contrast, in junctions where SPP excitation and associated light emission is driven by quantum mechanical tunneling, the drive voltages are low ( $<2.5 \mathrm{~V}$ for visible light), current-voltage, $I(V)$, characteristics are parabolic, and the light emission spectra blue-shifts with increasing bias, with the upper limit defined by the quantum cutoff law. ${ }^{45}$ To date, no MISJs have been reported that show these characteristics.

Watanabe et al. ${ }^{27}$ observed visible light emission from an $\mathrm{Si}-\mathrm{SiO}_{2}-\mathrm{Au} / \mathrm{Ag} \mathrm{MISJ}$ at $6.5 \mathrm{~V}$, which they attribute to SPP excitation and scattering from residual surface roughness, although the doping concentration of the n-type Si electrode was not reported. Similarly, Wang et al. ${ }^{41}$ also applied a very large DC voltage bias $(5 \sim 7 \mathrm{~V})$ to $\mathrm{Si}-\mathrm{SiO}_{2}-\mathrm{Au}$ MISJs with $N_{\mathrm{d}}$ $=2.5 \times 10^{19} \mathrm{~cm}^{-3}$ and observed light emission attributed to decaying SPPs, however, they used a barrier thickness of 10 $\mathrm{nm}$, which is too thick for tunneling to occur and, therefore, the light emission likely involved dielectric breakdown. Göktas et al. ${ }^{33}$ observed light emission from MISJs with p-type silicon doped with $N_{\mathrm{d}}=2 \times 10^{17} \mathrm{~cm}^{-3}$ and proposed that tunneling of accumulated holes generated an MISJ cavity mode that outcoupled to interface SPPs, scattering to photons. This mechanism is unlikely given the low doping levels and lack of spectral blueshift characteristic of SPP excitation in tunneling junctions. As a group, these studies did not investigate the importance of the doping of the Si, but control over the doping levels is important for the following reasons: (i) MISJs with low doping levels in contact with $\mathrm{Au} / \mathrm{Ag}$ typically create Schottky diodes where the mechanism of charge transport across the barrier is thermionic emission, (ii) MISJs with low doping levels lack tunneling as the main charge transport mechanism and SPPs cannot be excited by inelastically tunnelling electrons, and (iii) MISJs with low doping have large depletion layers resulting in Schottky barriers that require high voltages for electroluminescence and, consequently, large device drive voltages. In other words, in MISJs with Si with low doping levels, the mechanism of charge transport is not dominated by quantum mechanical tunnelling, and light emission stems from electroluminescence. ${ }^{46}$

In junctions that are dominated by quantum mechanical tunneling, such as MIM-TJs, no depletion layers nor Schottky barriers are present, and the charge carriers tunnel across the barrier elastically. In MIM-TJs with plasmonically active metals at visible wavelengths, for example, $\mathrm{Au}$ or $\mathrm{Ag}$, the excitation of the highly confined SPP mode inside the MIM-TJ cavity (the MIM-SPP mode) from inelastic tunneling can be as high as $10 \%$, which can lead to light emission via scattering. ${ }^{47}$ For plasmonically active tunnel junctions sustaining sufficiently high tunnel currents $(>100 \mu \mathrm{A})$, photon emission from the spontaneous emission of hot electron distribution has been extensively investigated in the past, where significant over bias emission is reported as a characteristic feature of the process. $^{48-51}$ Photons can also be directly excited from inelastic tunneling, especially in MIM-TJs where cavity modes cannot be supported. ${ }^{52,53}$ In principle, MISJs with highly doped semiconductors can both have charge carriers couple onto as well as support a hybrid-SPP mode inside the junction due to the presence of the low index $\mathrm{SiO}_{2}$ in between the high index $\mathrm{Si}$ and plasmonically active Au. ${ }^{54}$ In principle, this hybrid mode also offers opportunities to integrate MISJs with waveguides supporting hybrid modes for subwavelength field confinement along with a substantial increase in propagation length as compared to MIM-TJs. ${ }^{13-15}$

In systems dominated by thermionic emission, light emission is mainly due to electron-hole recombination, either from direct recombination of the hot electron or impact ionization where the energized electron causes a secondary electron-hole recombination during relaxation. ${ }^{17-23,28-32,34-40}$ In our $\mathrm{nSi}$ system (where $\mathrm{nSi}$ represents n-doped $\mathrm{Si}$ ), however, the current flow is from the $\mathrm{Si}$ (grounded) to the $\mathrm{Au}$ (biased) under a positive applied bias, so electron-hole recombination from thermionic emission is not favorable. ${ }^{46}$ However, at high enough applied bias, minority carrier injection into the $\mathrm{Si}$ is possible, which can lead to electron-hole recombination. ${ }^{55}$ Moreover, light emission from minority carrier injection at high bias is typically current independent and lacks a blueshift, with the energy of the emitted photons reflecting the bandgap of $\mathrm{Si}^{46}$ Thus, studying the light emission from MISJs with different $N_{\mathrm{d}}$, coupled with analyzing the charge transport mechanisms from the $I(V)$ curves and bias-dependent emission spectra, is 
needed to understand the plasmonic properties of such systems.

Here we report MISJs of the form $\mathrm{Au}-\mathrm{SiO}_{2}-\mathrm{nSi}$, with $\mathrm{N}_{\mathrm{d}}$ of $\mathrm{nSi}$ varying over 4 orders of magnitude (from $1.6 \times 10^{15}$ to 1.0 $\left.\times 10^{20} \mathrm{~cm}^{-3}\right)$. Only MISJs with the highest doping level $\left(N_{\mathrm{d}}=\right.$ $1.0 \times 10^{20} \mathrm{~cm}^{-3}$ ) have $I(V)$ characteristics indicating that quantum mechanical tunneling dominates the mechanism of charge transport, and both photons and SPPs can be excited by the inelastic electron energy loss at low applied voltages $(V<2$ V). MISJs with moderately high doping $\left(N_{\mathrm{d}}=10^{18} \mathrm{~cm}^{-3}\right)$ have $I(V)$ characteristics that show a mix of Schottky diode behavior and quantum tunneling, with light emission observable over a bias window that extends beyond the breakdown seen in purely quantum system $(1.5 \mathrm{~V}<V<2.5 \mathrm{~V})$. MISJs with moderate doping $\left(N_{\mathrm{d}}=10^{15}-10^{16} \mathrm{~cm}^{-3}\right)$ have $I(V)$ characteristics of Schottky diodes, and light emission is only observable at high bias $(V>5.5 \mathrm{~V})$ due to higher barrier width (reaching the field emission limit), and the optical spectra are dominated by photons generated via electroluminescence. MISJs with the lowest doping level $\left(N_{\mathrm{d}}=1.6 \times 10^{15} \mathrm{~cm}^{-3}\right)$ did not emit light within the detection limit of our setup. To demonstrate the quantum mechanical tunneling excitation of the hybrid-SPP modes in highly doped MISJs, we analytically calculated the LDOS of the MISJ and corroborated the electromagnetic response of the MISJ using FEM.

\section{RESULTS AND DISCUSSIONS}

Mechanism of Charge Transport across MISJs. Current flow across an MISJ depends on the insulator thickness $d$ (in $\AA$ ), depletion layer width $W_{\mathrm{D}}$, and $N_{\mathrm{d}}$, and is dominated by either (a) electron flow over the potential barrier from the semiconductor to the metal (thermionic emission) or (b) quantum mechanical tunneling, depending on $N_{\mathrm{d}}$. Due to the presence of the oxide layer as an additional tunneling barrier along with the built-in field caused by the depletion layer, the net current $I$ across the barrier can be written as a combination of tunneling and charge transport over a Schottky barrier: ${ }^{46,56}$

$$
I=I_{0} \exp (-\sqrt{\zeta} d) \exp \left(-\frac{q \phi_{B n_{0}}}{k_{\mathrm{B}} T}\right)\left(\exp \left(\frac{q\left(V-I R_{\mathrm{S}}\right)}{\eta k_{\mathrm{B}} T}\right)-1\right)
$$

where $\zeta$ is the tunneling barrier height (in $\mathrm{eV}$ ). The term $\exp (-\sqrt{\zeta} d)$ represents the tunneling probability across the insulator, $\phi_{B n_{0}}$ is the Schottky barrier height, $q$ is the electronic charge, $k_{\mathrm{B}}$ is the Boltzmann's constant, $T$ is the temperature, $R_{\mathrm{S}}$ represents the junction series resistance, and $\eta$ gives the ideality factor (eq 2). The pre-exponential factor $I_{0}=A_{\mathrm{j}} \times$ $A^{* *} T^{2}$, where $A^{* *}$ is the effective Richardson constant and $A_{\mathrm{j}}$ is the junction area.

Figure 1a shows the energy level diagram under forward bias for an $\mathrm{Au}-\mathrm{SiO}_{2}-\mathrm{nSi}$ junction with moderately doped $\mathrm{nSi}\left(N_{\mathrm{d}}\right.$ $\left.\sim 10^{15}-10^{16} \mathrm{~cm}^{-3}\right)$. The potential is described on the Au side by the Au work function $\phi_{\mathrm{Au}}, \Xi$ gives the potential of the interface layer and the Si potential is defined by the electron affinity of the Si $\chi$ at the barrier, where $\psi_{i}$ defines the built-in potential of the $\operatorname{Si} . \varepsilon_{\mathrm{c}}$ and $\varepsilon_{\mathrm{v}}$ give the conduction and valence band of the $\mathrm{nSi}$, respectively, with $\varepsilon_{\mathrm{f}}^{\mathrm{Si}}$ and $\varepsilon_{\mathrm{f}}^{\mathrm{Au}}$ giving the Fermi energies of the $\mathrm{nSi}$ and $\mathrm{Au}$, respectively. Figure $1 \mathrm{~b}$ shows the energy level diagram at forward bias for an MISJ system for the highly doped case $\left(N_{\mathrm{d}} \sim 10^{20} \mathrm{~cm}^{-3}\right)$, where band bending is negligible. It is expected that, in these systems, $\psi_{i} \rightarrow 0$ and $\chi$ can be described by an effective work function of the Si $\phi_{\mathrm{Si}}$ (inset to Figure $1 \mathrm{~b}$ ), where $W_{\mathrm{D}}$ is on the order of a few nanometers (see the next section). Crucially, charge transport in these systems will be dominated by quantum mechanical tunneling and have symmetrical $I(V)$ properties. This qualitative description is further confirmed by the depletion width calculation followed from the experimental currentvoltage characteristics (see eq 3 and Figure 3f).

Assuming negligible interface states (any intermediate energy states in the barrier caused by charge traps, dangling bonds, etc.), the $\eta$ is related to the depletion width $W_{\mathrm{D}}$ as ${ }^{57}$

$$
\eta=1+\frac{d \epsilon_{\mathrm{Si}}}{W_{\mathrm{D}} \epsilon_{1}}
$$

where $\epsilon_{\mathrm{Si}}$ and $\epsilon_{1}$ represent the dielectric constants of $\mathrm{nSi}$ and $\mathrm{SiO}_{2}$, respectively. We follow Cheung's method ${ }^{56}$ to extract the value of $\eta$ from the forward $I(V)$ characteristics and use eq 2 to calculate the $W_{\mathrm{D}}$ as a function of $N_{\mathrm{d}}$, as described below. Rearranging eq 1 gives

$$
\frac{\mathrm{d} V}{\mathrm{~d}(\ln J)}=A J R_{\mathrm{S}}+\frac{\eta k_{\mathrm{B}} T}{q}
$$

where $J$ represents the total current density. A linear fit of a plot of $\frac{\mathrm{d} V}{\mathrm{~d}(\ln J)}$ versus $J$ to eq 3 gives $\eta$ from the $y$-intercept. Understanding the behavior of the effective barrier width (which includes the depletion layer) as a function of $N_{\mathrm{d}}$ is of vital importance in the present analysis. In general, for a given bias, inelastic tunneling across the insulator layer and the subsequent SPP excitation probability depends on the effective barrier width and, because of the hybrid plasmonic nature of the junction in our systems, it also depends on $N_{\mathrm{d}}$ of the semiconductor. We note that the experimental spectra reported here elucidates this doping level dependency on the light emission/SPP excitation explicitly (see Figure 4).

Plasmon Excitation Mechanism in MISJs. As discussed earlier, SPP excitation is the predominant decay channel when charge transport is dominated by quantum tunneling. For an MISJ in the limit of $W_{\mathrm{D}} \rightarrow 0$ (highly doped system), we calculated the power dissipated by a dipole emitter located at the middle of the insulator layer ${ }^{58}$ to obtain the LDOS of the MISJ (Figure 2a) and the mode dispersion relation (Figure 2b; see Supporting Information, Section S7 for more information). A static relative permittivity $\epsilon_{\mathrm{Si}}=11.7$ is assigned for the $\mathrm{nSi}$ electrode in the calculation with negligible doping dependence on the permittivity in the investigated energy range ${ }^{59}$ (as shown by the ellipsometry data in Figure S3) and free-electron Drude model is used to represent the Au electrode. ${ }^{60}$ Figure $2 \mathrm{a}$ shows the normalized power dissipation $p$ as a function of $k$ / $k_{0}$, where $k$ is the in-plane wavevector and $k_{0}$ represents the free space wavevector corresponding to the energy $\sim 1.5 \mathrm{eV}$. Due to the thin $\mathrm{SiO}_{2}$ layer on top of the $\mathrm{nSi}$, the SPP excited at the $\mathrm{Au}-\mathrm{SiO}_{2}-\mathrm{nSi}$ interface has its dispersion characterized by the electromagnetic properties of both the $\mathrm{SiO}_{2}$ and $\mathrm{nSi}$; this mode is most commonly termed a hybrid-SPP mode. The main sharp peak at $k / k_{0} \approx 4$ represents the hybrid-SPP mode supported by the junction (Figure 2a). Since the $n S i$ wafer is opaque at optical frequencies light emission measurements where recorded from the $\mathrm{Au}$ electrode side with an oil immersion objective (refractive index $=1.515$ ) and the small peak at $k / k_{0} \approx 1.5$ represents the $\mathrm{Au}$-oil single interface SPP mode supported in this configuration. 


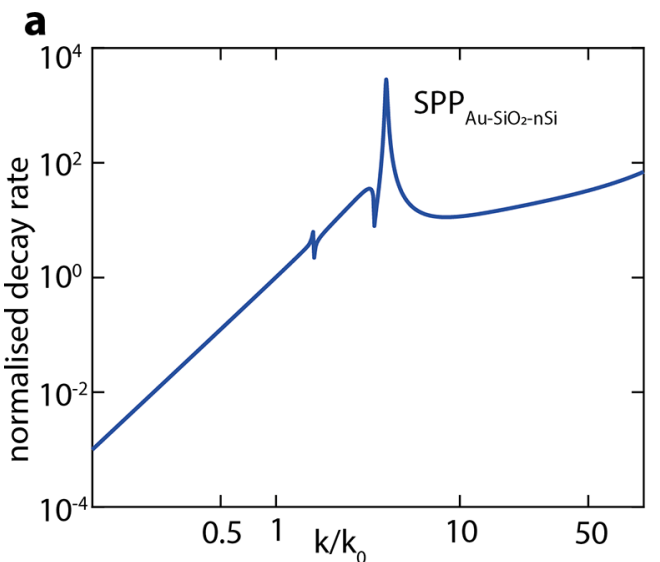

b

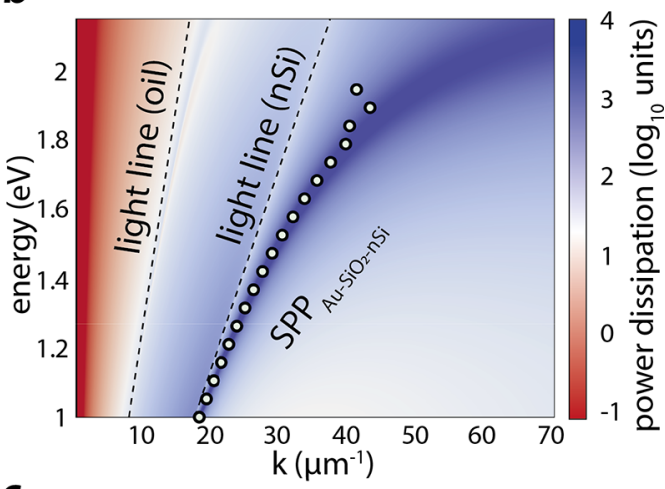

C

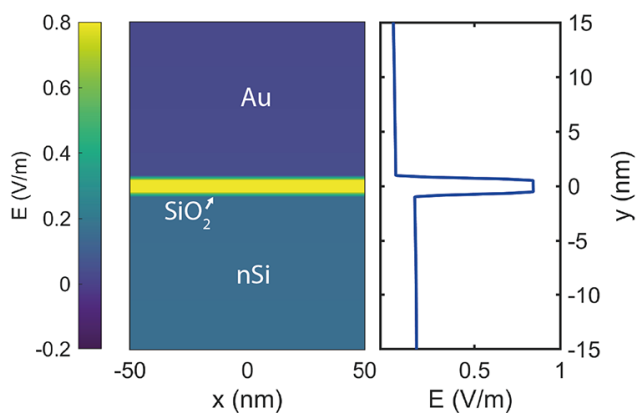

Figure 2. (a) Dipole power dissipation evaluated for $\mathrm{Au}-\mathrm{SiO}_{2}-\mathrm{nSi}$ junction at $1.5 \mathrm{eV} . \epsilon_{\mathrm{Si}}=11.7, \epsilon_{1}=2.1$, and Drude model for Au top electrode (50 nm thickness). (b) Dipole power dissipation evaluated as a function energy and wavevector, representing the dispersion for the hybrid-SPP mode. Open circles: mode index of the hybrid-SPP mode obtained from the FEM mode analysis. Dashed lines: light lines in oil and $\mathrm{nSi}$. (c) Normalized electric field (E) profile (left) of the MISJ at $1.5 \mathrm{eV}$ obtained from the FEM model with cross-section line profile at $x=0$ (right) showing the high field confinement in the $\mathrm{SiO}_{2}$ layer.

As mentioned in the first section briefly, the presence of a low index $\left(\mathrm{SiO}_{2}\right)$ layer between a high index $\mathrm{nSi}$ and a plasmonic metal $(\mathrm{Au})$ defines the hybrid nature of the mode supported by the MISJ and makes it highly confined within the insulator layer (Figure 2c). This confinement is evident from the large normalized power dissipation $\left(p \sim 10^{3}\right)$ associated with the hybrid-SPP mode in comparison with the singleinterface SPP mode $\left(p \sim 10^{1}\right)$ supported by the Au-oil interface (Figure 2b). This high LDOS associated with the hybrid-SPP mode effectively enhances the probability of SPP excitation (with energy $\hbar \omega$ ) in the inelastic electron tunneling process as observed in typical MIM-TJs.
We performed FEM mode analysis with COMSOL Multiphysics $^{61}$ to further confirm the highly confined nature of the hybrid-SPP mode (Figure 2c, see Supporting Information, Section S7 for more information). The heatmap in Figure 2c (left) represents the normalized electric field intensity of the hybrid-SPP mode supported by the MISJ and the line profile (right) shows the relative intensity in the respective layers. As evident from the line profile, the maximum field intensity is localized within the $2 \mathrm{~nm}$ thick $\mathrm{SiO}_{2}$ layer. We also compare the mode index of the hybrid-SPP mode obtained from the FEM model with LDOS calculations, which is in excellent agreement with the analytical results (open circles in Figure 2b). As the energy approaches the interband transition energy of $\mathrm{Au}(>2 \mathrm{eV})$, the effective mode index obtained from FEM starts to deviate from the hybridSPP mode in the dispersion plot (Figure 2b). It should be noted that the presence of an SPP mode with high LDOS is essential for the inelastic tunneling process to efficiently excite SPPs. This analysis quantifies the LDOS associated with hybrid-SPP mode which is further elucidated in the experimental emission spectra from MISJs recorded as a function of doping concentration discussed below.

Electrical Characterization of the Junctions. We fabricated chips of $\mathrm{Au}-\mathrm{SiO}_{2}-\mathrm{nSi}$ junctions with four different $N_{\mathrm{d}}$ values (see Table 1) with a $2.8 \pm 0.1 \mathrm{~nm}$ thick $\mathrm{SiO}_{2}$

Table 1. List of Silicon Substrates Used with Resistivity and Doping Concentration

\begin{tabular}{cccc} 
type & dopant & resistivity $(\Omega \cdot \mathrm{cm})$ & \multicolumn{1}{c}{$N_{\mathrm{d}}\left(\mathrm{cm}^{-3}\right)$} \\
$\mathrm{n}$ & As & $0.001-0.005$ & $1.2 \times 10^{19}$ to $9.7 \times 10^{19}$ \\
$\mathrm{n}$ & $\mathrm{Sb}$ & $0.01-0.02$ & $1.2 \times 10^{18}$ to $3.6 \times 10^{18}$ \\
$\mathrm{n}$ & $\mathrm{P}$ & $0.3-0.5$ & $1.1 \times 10^{16}-1.9 \times 10^{16}$ \\
$\mathrm{n}$ & $\mathrm{P}$ & $3-9$ & $5.0 \times 10^{14}$ to $1.6 \times 10^{15}$ \\
\hline
\end{tabular}

(measured with ellipsometry, Figure S3) layer and $62 \mathrm{~nm}$ thick $\mathrm{Au}$ top electrodes. Each chip consisted of a $6 \times 6$ array of junctions each with an area of $5 \times 5 \mu \mathrm{m}^{2}$. Section $S 1$ describes the fabrication in detail. Briefly, we formed an $81 \mathrm{~nm}$ layer of $\mathrm{SiO}_{2}$ on freshly cleaned $\mathrm{Si}$. After patterning, the $\mathrm{SiO}_{2}$ layers were removed and an ultrathin $\mathrm{SiO}_{2}$ tunneling barrier was thermally grown. Finally, the Au top electrode was deposited giving an isolated MISJ. A schematic of the MISJ is given in Figure $3 \mathrm{a}$ along with the thickness of each layer indicated in the inset, and a false-color scanning electron microscope (SEM) image is shown Figure 3b. Figure S4 shows an example of a packaged array of the junctions used in our experiments.

Figure 3c shows the $I(V)$ curve for each type of device where junctions with the highest value of $N_{\mathrm{d}}$ have a symmetrical $I(V)$ curve associated with quantum mechanical tunneling, while the $I(V)$ curves become increasingly more asymmetrical with decreasing $N_{\mathrm{d}}$ typically observed for Schottky diodes. ${ }^{7,8,62}$ This asymmetry can be quantified by taking the ratio of the current at two reciprocal voltage values, that is, the rectification ratio $R_{\mathrm{r}}$ $=I(+1 \mathrm{~V}) / I(-1 \mathrm{~V})$. Figure $3 \mathrm{~d}$ (right axis) shows that as $N_{\mathrm{d}}$ increases, the value of $R_{\mathrm{r}}$ decreases from $10^{3}$ for $N_{\mathrm{d}} \approx 1 \times 10^{15}$ $\mathrm{cm}^{-3}$, to unity for $N_{\mathrm{d}} \approx 1 \times 10^{20} \mathrm{~cm}^{-3}$, implying that $W_{\mathrm{D}}$ decreases for increasing $N_{\mathrm{d}}$ (as shown in Figure 1). This reduction in $W_{\mathrm{D}}$ raises the zero bias conductance and associated innate conductivity of the system in the steadystate with no applied bias (Figure $3 \mathrm{~d}$; left axis). In other words, the tunneling barrier becomes increasingly square, signaling a transition to quantum tunneling dominated charge transport. 

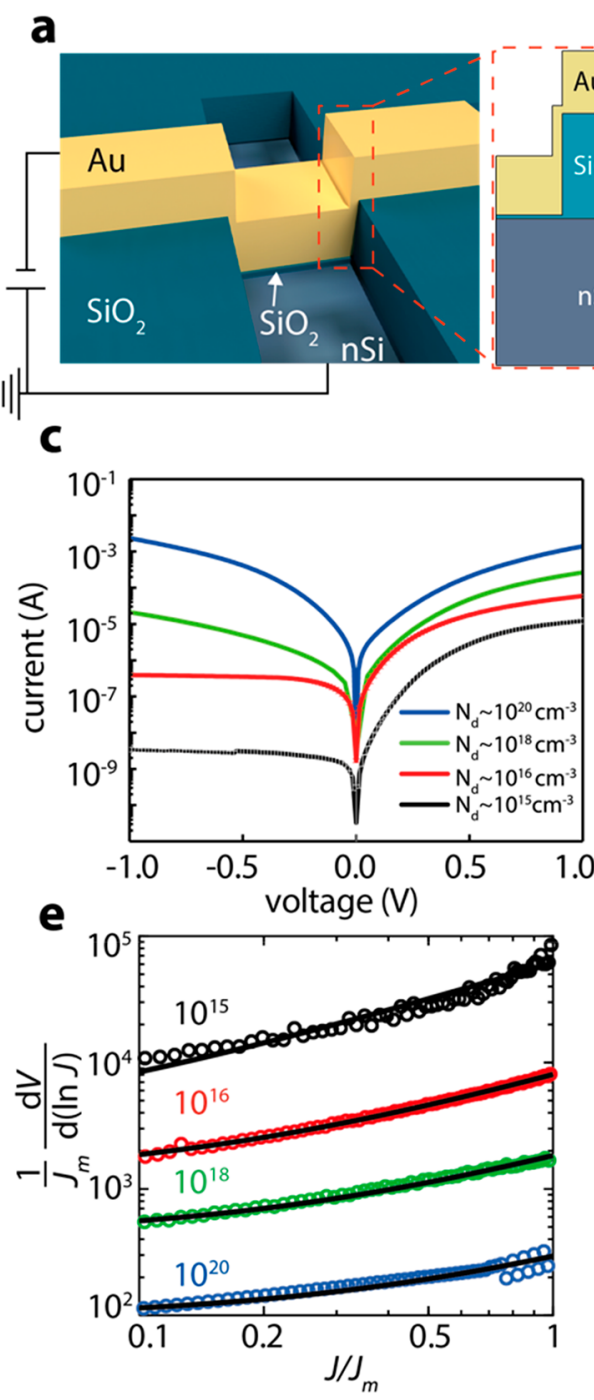

b
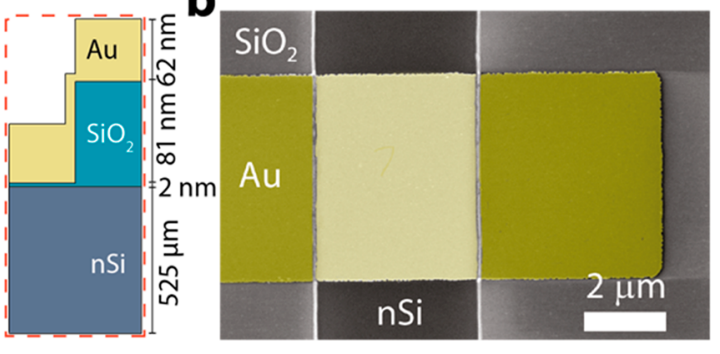

d
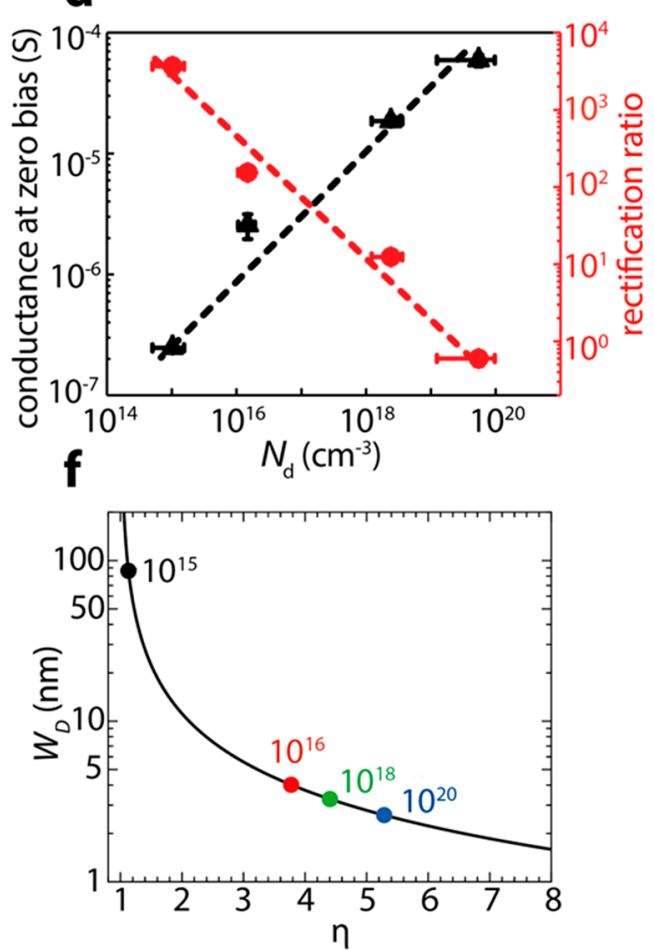

Figure 3. (a) Schematic of the devices along with a cross section (inset) indicated by the dashed box. (b) False color SEM image of a device to distinguish the Au electrode (the unmodified image is shown in Figure S2). (c) I(V) plots recorded from the MISJs with the different doping levels indicated. (d) The conductance at zero bias plotted on the left axis (black triangles) and rectification ratio on the right axis (red circles). Dashed lines are visual guides. The error bars represent standard deviation from five $I(V)$ scans. (e) $\mathrm{d} V / \mathrm{d}(\ln J)$ evaluated using eq 3 , from the $I(V)$ data in (c), for each value of $N_{\mathrm{d}}$ plotted as a function of $J$ (open circles). The axes are rescaled by the maximum current density $J_{\mathrm{m}}$ from the corresponding data to improve clarity. Solid line shows the fit to eq 3. (f) $W_{\mathrm{D}}$ as a function of $\eta$ obtained from the fitting result shown in Figure $3 \mathrm{e}$ (solid circles). For $\eta$ in the range of 1 to $8, W_{\mathrm{D}}$ calculated from eq 2 using $d=2 \mathrm{~nm}, \epsilon_{\mathrm{Si}}=11.7$, and $\epsilon_{1}=2.1$ is also plotted (solid line) to show the variation in $W_{\mathrm{D}}$. Each doping level is indicated in $\mathrm{cm}^{-3}$.

From the experimental $I(V)$ data shown in Figure $3 c, d V /$ $\mathrm{d}(\ln J)$ is evaluated for each value of $N_{\mathrm{d}}$ and is plotted as a function of current density $J$ in Figure $3 e$ (open circles). The axes are rescaled by the maximum current density $J_{\mathrm{m}}$ from the corresponding data to improve clarity. From eq 3, variation in $\mathrm{d} V / \mathrm{d}(\ln J)$ as a function of $J$ can be fitted to a straight line (solid lines in Figure 3e) and from the $y$-intercept of the fit $\eta$ can be directly obtained (see eq 3 ). From $\eta$ obtained for each $N_{\mathrm{d}}$ value and using eq 2, the reduction of $W_{\mathrm{D}}$ with increasing $N_{\mathrm{d}}$ can be quantitatively estimated. Figure $3 \mathrm{f}$ shows $W_{\mathrm{D}}$ (solid circles) calculated from eq 2 using $\eta$ obtained for each $N_{\mathrm{d}}$. The solid line in Figure $3 \mathrm{f}$ shows a fit to eq 2 with $W_{\mathrm{D}}$ as a function of $\eta$, calculated using $d=2 \mathrm{~nm}, \epsilon_{\mathrm{Si}}=11.7$, and $\epsilon_{1}=2.1$, for $\eta$ in the range of 1 to 8 . This plot shows that $W_{\mathrm{D}}$ decreases from $\sim 100$ to $\sim 2 \mathrm{~nm}$ with an increase in $N_{\mathrm{d}}$ from $N_{\mathrm{d}} \sim 10^{15} \mathrm{~cm}^{-3}$ to $N_{\mathrm{d}} \sim 10^{20} \mathrm{~cm}^{-3}$. This transition to a narrow depletion layer, with significantly reduced band-bending, confirms the transition from Schottky (Figure 1a) to tunneling (Figure 1b) behavior. Figure $3 \mathrm{f}$ shows that $\eta$ increases from $\sim 1$ (pure Schottky diode behavior) to $\sim 5$ (tunneling behavior) ${ }^{63}$ corroborates the transition of the charge transport mechanism from thermionic emission to tunneling.

Optical Properties of the Junctions. We measured the optical light emission from all devices using an electronmultiplying CCD (EMCCD) camera following previously described methods (see Supporting Information, Section S5 $)^{7,8}$ from the Au electrode side. Three of the four MISJs emitted light under the applied voltage range, but junctions with $N_{\mathrm{d}}=2 \times 10^{15} \mathrm{~cm}^{-3}$ did not emit enough light to be measurable with our setup (in the bias range from 1 to $10 \mathrm{~V}$ ). Figure $4 a-c$ shows the EMCCD images of the three junctions that emitted light, with the applied bias voltage indicated and intensity scaled to give best contrast. 
a

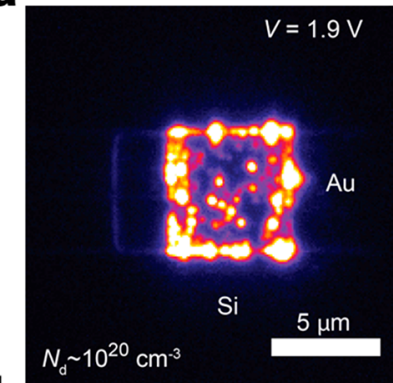

b

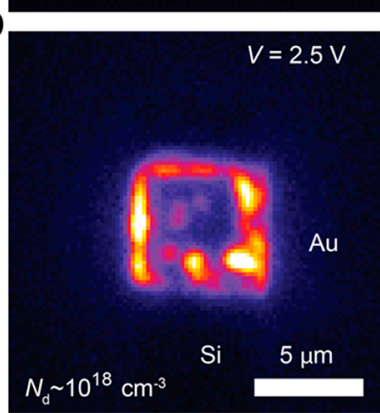

C

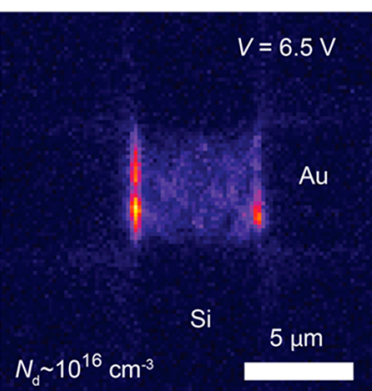

d

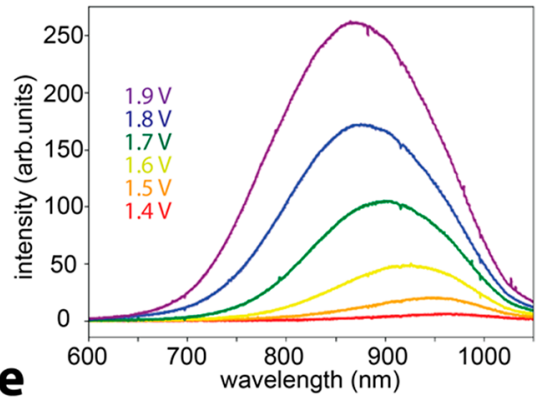

e

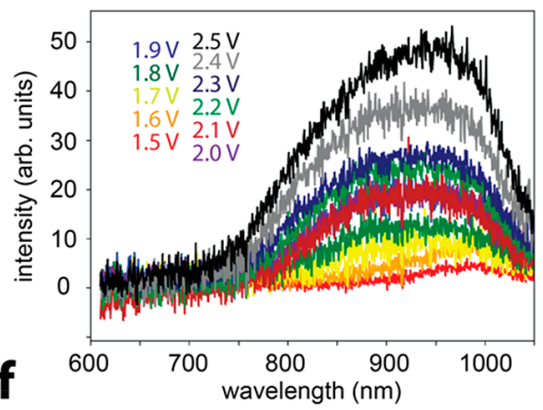

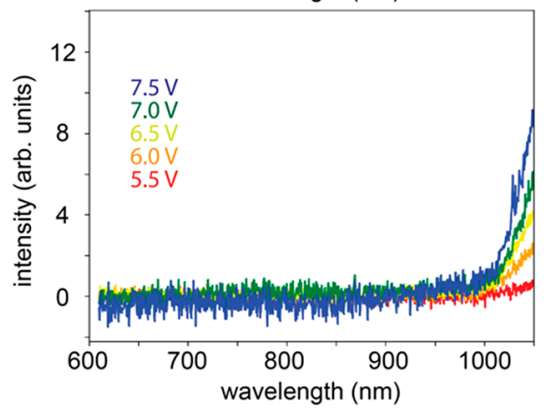

Figure 4. (a-c) EMCCD images obtained from the junctions for (a) $N_{\mathrm{d}} \sim 1 \times 10^{20} \mathrm{~cm}^{-3}$ at $1.9 \mathrm{~V}$, (b) $N_{\mathrm{d}} \sim 4 \times 10^{18} \mathrm{~cm}^{-3}$ at $2.5 \mathrm{~V}$, and (c) $N_{\mathrm{d}} \sim 2$ $\times 10^{16} \mathrm{~cm}^{-3}$ at $6.5 \mathrm{~V}$. (d-f) Corresponding spectra for a range of measured voltages.

Notably, light emission across the MISJ area increases with increasing $N_{\mathrm{d}}$, which can be explained as follows. In junctions with large $N_{\mathrm{d}}$, the SPPs excited inside the MISJs can scatter off of defects induced by the surface roughness of the electrodes from anywhere in the MISJ area, as demonstrated in our recent works. ${ }^{6,8} \mathrm{We}$ also estimated an electron-to-photon conversion efficiency of roughly 1 photon in $10^{9}$ electrons for our MISJs, which is $\sim 2$ orders of magnitude lower than the MIM junctions reported in our previous works ${ }^{7,8}$ and that of other studies $^{4,45}$ computed in the same way; this relatively low efficiency can be explained as follows. The electron-to-photon conversion efficiency in MIM junctions is significantly improved by the electrode roughness ${ }^{6}$ (and, therefore, electrode roughening has been used in the past ${ }^{5}$ ), which is minimal in our case due to the presence of a smooth $\mathrm{Au}-\mathrm{SiO}_{2}$ $\mathrm{nSi}$ interface. Moreover, the relatively thick Au top electrode (62 nm) causes substantial electromagnetic screening, which further reduces the effective photon outcoupling to the far-field (as described in ref 8 ) of MISJs reported here. For light emission by electron-hole recombination for junctions with low $N_{\mathrm{d}}$, the photons have to pass through the $62 \mathrm{~nm}$ thick $\mathrm{Au}$ top electrode, except at the edges of the MISJ, which explains why light emission dominates at the junction edges in Figure $4 c$.

Figure $4 \mathrm{~d}-\mathrm{f}$ shows the spectral response of the MISJs and the redshift of the main peak with increasing $N_{\mathrm{d}}$. For the junctions with $N_{\mathrm{d}} \sim 1 \times 10^{20} \mathrm{~cm}^{-3}$, light emission is detectable as low as $1.4 \mathrm{~V}$, the light emission intensity increases with bias, and the spectra blueshifts (Figures $4 \mathrm{~d}$ and $5 \mathrm{a}, \mathrm{b}$ ) with bias up to $1.9 \mathrm{~V}$ (the junctions irreversibly broke down at large applied voltages). For the intermediate case with $N_{\mathrm{d}} \sim 4 \times 10^{18} \mathrm{~cm}^{-3}$, light emission is detectable at a similarly low voltage of $1.5 \mathrm{~V}$, but only a small blueshift with increasing voltage up to $2.5 \mathrm{~V}$ (Figures $4 \mathrm{e}$ and $5 \mathrm{a}, \mathrm{b}$ ) is observed (the junctions broke down at large voltages). For junctions with $N_{\mathrm{d}} \sim 2 \times 10^{16} \mathrm{~cm}^{-3}$, however, there is no detectable emission until $5.5 \mathrm{~V}$, and even at this point the signal is significantly red-shifted that it falls mainly outside of the detectable range of our Si-based spectrometer of up to $\sim 1050 \mathrm{~nm}$ (Figure $4 \mathrm{f}$ ). These results can be interpreted as follows. For light emission from the roughness-scattered hybrid-SPP mode, the spectral peak position should blueshift according to the increasing applied bias, with the upper limit of the front edge of the spectral peak defined by the quantum cutoff $\hbar \omega \leq \mathrm{leVl}$ (see Figure 5 for a full characterization), which is observed for $N_{d} \sim 1 \times 10^{20}$ $\mathrm{cm}^{-3}$ (Figure 4d), with the MISJ breaking down well below the interband transition energy of $\mathrm{Au}(2.2 \mathrm{~V})$. These results indicate that light emission originates from radiative decay of SPPs. A similar but less profound blueshift is observed for $N_{\mathrm{d}}$ $\sim 4 \times 10^{18} \mathrm{~cm}^{-3}$ (Figure 4e) at slightly higher voltages, which suggests the emitted light is not entirely SPP dominated. In contrast, for light emission from systems where thermionic 
a
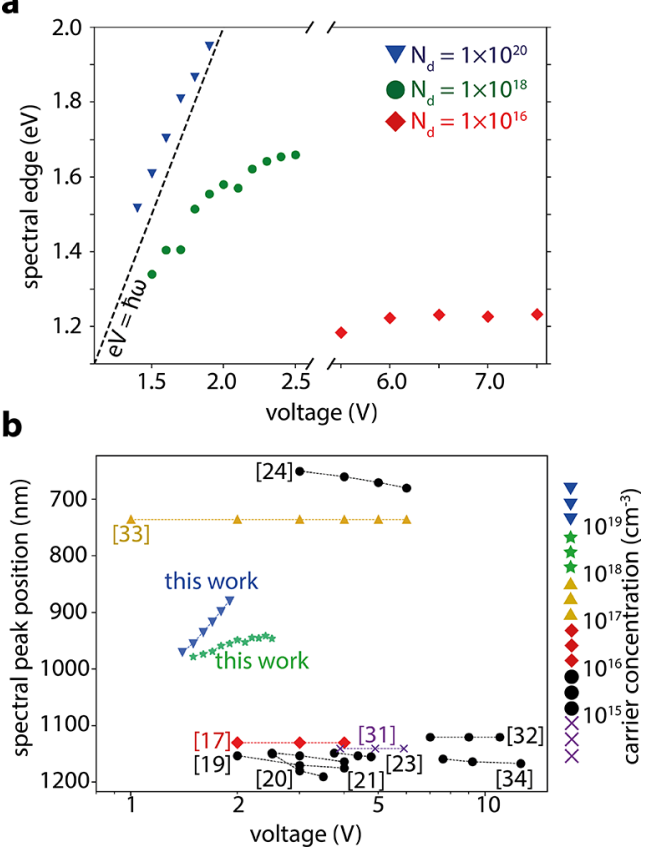

Figure 5. (a) Front spectral edge of the spectra from each device in Figure 4, plotted in $\mathrm{eV}$ and compared to the quantum cutoff (black dashed line) with each $N_{\mathrm{d}}$ value labeled. (b) Spectral peak shift as a function of $V$, color coded by $N_{d}$ for literature values (the corresponding references are indicated in the square brackets) that report spectra as a function of at least three values of $V$ for MISJs.

emission dominates the mechanism of charge transport, the spectra lack the spectral shift as a function of $V$, with the energy of the emitted light close to the band gap of the semiconductor (and thus far from the energy of the quantum cutoff). In case of the junction with $N_{\mathrm{d}} \sim 2 \times 10^{16} \mathrm{~cm}^{-3}$, the energy of the emitted light is far below the quantum cutoff and close in energy to that of the bandgap of Si. Since silicon is not an efficient electroluminescent material, overall light emission is weak.

To determine the transition from junctions dominated by quantum mechanical tunneling to those dominated by electroluminescence, we qualitatively analyze and spectrometry data in Figure 5. Figure 5a shows the front edge of each spectra in $\mathrm{eV}$ (see Section S6 for how the front edge was determined) plotted for each $N_{\mathrm{d}}$, all against the quantum cutoff denoted by the black dashed line. For $N_{\mathrm{d}}=1 \times 10^{20} \mathrm{~cm}^{-3}$ case, emission follows the quantum cutoff law $(\mathrm{leVl}=\hbar \omega)$ linearly, with a nearly constant overbias contribution $(\sim+100 \mathrm{meV})$ within the applied bias range, which we attribute to the hot electrons in the system. ${ }^{51}$ It is clear that as $N_{\mathrm{d}}$ decreases, the adherence to the quantum cutoff diminishes. The spectra recorded from junctions with $N_{\mathrm{d}}=2 \times 10^{16} \mathrm{~cm}^{-3}$ (Figure 5a) lack a clear peak and hence no correlation could be determined in this case.

In principle, the light emission intensity decreases as a function of $W_{\mathrm{D}}$ since the charge carriers have to traverse an increasingly larger distances, causing the minimum voltage needed to detect light emission to increase. In addition, as $N_{\mathrm{d}}$ decreases, the minimum voltage at which light emission is detectable increases due to the decrease in inelastic tunneling rate. Minority carrier injection requires much higher voltages, which can be seen from the different voltage dependences as a function of $N_{\mathrm{d}}$ in both Figures 4 and 5. For a purely thermionic system with $N_{\mathrm{d}} \sim 2 \times 10^{16} \mathrm{~cm}^{-3}$, light emission is not observable until $V=5.5 \mathrm{~V}$ (Figure 4c, comparable with other studies of light emission from minority carrier injection ${ }^{55}$ ). We were unable to detect light emission from junctions with the lowest $N_{\mathrm{d}} \sim 2 \times 10^{15} \mathrm{~cm}^{-3}$, where the minority carrier injection rate under forward bias is not high enough to induce enough interband transitions to allow electron-hole recombination because of the very large $W_{\mathrm{D}} \sim 80 \mathrm{~nm}$ (Figure 3f).

Comparing our results to the wider body of literature enables us to resolve discrepancies in the explanations regarding light emission from MISJs. Figure $5 b$ shows the spectral peak positions as a function of $V$ from the literature (we only included reports that report at least three spectra at three different voltage values) and have color coded each data set with the reported $N_{\mathrm{d}}$ value. The majority of MISJ literature focuses on MISJs, with $1 \times 10^{14} \mathrm{~cm}^{-3}<N_{\mathrm{d}}<1 \times 10^{16} \mathrm{~cm}^{-3}$ (purple and black symbols in Figure 5b), and all (except for two reports) describe junctions with light emission in the NIR region and conclude that light emission is the result of electron-hole recombination. Ref 24 suggests that the visible light emission is due to luminescent centers caused by defects and impurities (mainly oxygen vacancies) in the $\mathrm{SiO}_{2}$ film in their devices. In ref 33, Göktas and colleagues propose that SPP excitation and scattering is the dominant light emission mechanism, but for the following three reasons, we disagree with their interpretation: (i) the light emission in their system lacks the spectral peak shift as a function of $V$, (ii) their given $I(V)$ curves show very clear rectification, and (iii) their light emission intensity is far away from the quantum cutoff regime. These observations indicate that their junctions are dominated by thermionic emission and associated electroluminescence. However, the only discrepancy in their results is the visible nature of the emitted light, which may be due to defects in their junction following the same explanation as in Ran et al. ${ }^{24}$ In contrast, only junctions with $N_{\mathrm{d}}=1 \times 10^{20} \mathrm{~cm}^{-3}$ show the characteristic spectral peak shift (the blue data set in Figure $5 \mathrm{~b}$ ), while junctions with $N_{\mathrm{d}}=4 \times 10^{18} \mathrm{~cm}^{-3}$ already deviate significantly from quantum cutoff (green data set in Figure $5 b$ ).

\section{CONCLUSIONS}

Here, we have experimentally shown that plasmons can be excited in $\mathrm{Au}-\mathrm{SiO}_{2}-\mathrm{nSi}$ MISJs by inelastic electron tunneling with a doping concentration of $1.0 \times 10^{20} \mathrm{~cm}^{-3}$ at a low applied voltage bias of $>1.4 \mathrm{~V}$. We have examined light emission from MISJs of different $N_{\mathrm{d}}$ and find that when $N_{\mathrm{d}} \sim 1$ $\times 10^{16} \mathrm{~cm}^{-3}$, the conduction is dominated by thermionic emission and there is no significant SPP excitation which is corroborated by a lack of a voltage dependency of the spectral peak position of the collected light emission. In contrast, when $N_{\mathrm{d}} \sim 1 \times 10^{20} \mathrm{~cm}^{-3}$, the charge transport is dominated by quantum mechanical tunneling, where inelastically tunneling electrons excite SPPs, resulting in a voltage-dependent spectral peak blueshift. We placed our results in context of light emission from other types of MISJs, and find that our results agree with the majority of works reported: light emission from junctions with low doping levels is dominated by electronhole pair recombination from minority carrier injection. In contrast, Göktas and colleagues ${ }^{33}$ claim SPP excitation as the dominant light emission mechanism in systems doped to $N_{\mathrm{d}} \sim$ $1 \times 10^{18} \mathrm{~cm}^{-3}$, but they do not see any spectral blue shift with the applied bias, and the electrical characterization of their junctions show typical Schottky characteristics. Based on the findings we report here, we believe that their results can be 
explained by standard thermionic emission and light emission due to defects in the barrier (such as oxygen vacancies), resulting in luminescence centers, as suggested by Ran et al. ${ }^{24}$

Our simulations show that the conductivity of the $\mathrm{Si}$ electrode in an $\mathrm{Au}-\mathrm{SiO}_{2}-\mathrm{nSi}$ MISJ has to be sufficiently high $\left(N_{\mathrm{d}}>1 \times 10^{19} \mathrm{~cm}^{-3}\right)$ to support quantum mechanical tunneling and subsequent SPP excitation. Our work paves the way for interesting future directions since MISJs based on $\mathrm{Si}$ can, in principle, be readily integrated with $\mathrm{Si}$-photonics platforms and connected to waveguides that support a variety of photonic or hybrid-SPP modes. Moreover, this work also indicates that SPP excitation from inelastic tunneling can be realized in a variety of materials and geometries, provided that the LDOS of the system supports an SPP mode, which gives much more flexibility to tunneling junction-based systems across nanocircuitry than have been currently realized.

\section{EXPERIMENTAL DETAILS}

Device Fabrication. Silicon substrates with an area of 1.5 $\mathrm{cm} \times 1.5 \mathrm{~cm}$ were immersed in acetone solution followed by isopropyl alcohol (IPA) to remove the organic residues on the silicon substrate. The native oxide was removed by buffered oxide etch (BOE) for $10 \mathrm{~s}$. The thermal $\mathrm{SiO}_{2}$ of thickness of $2.8 \mathrm{~nm} \pm 0.1 \mathrm{~nm}$ was grown in an oxidation furnace in air environment for $2 \mathrm{~h}$ at $800{ }^{\circ} \mathrm{C}$.

A three-step of lithography process was used to fabricate the $\mathrm{Au}-\mathrm{SiO}_{2}-\mathrm{nSi}$ junctions. The first step of lithography is employed to define the junction area on the $\mathrm{SiO}_{2} / \mathrm{nSi}$ substrate. A layer of $\mathrm{SiO}_{2}$ film with a thickness of $\sim 100 \mathrm{~nm}$ was deposited by RF magnetron sputtering. The samples were then immersed in acetone at room temperature overnight to lift-off the photoresist, leaving the junction area on the $\mathrm{Si}$ substrate. $\mathrm{Ti} / \mathrm{Au}$ pad contact electrodes were patterned and deposited by thermal evaporation. The junction area and waveguides were defined by electron beam lithography (EBL) followed by thermal evaporation.

Device Measurements. The current-voltage characteristics were measured to distinguish electron tunneling behavior and Schottky thermionic emission. Voltages were applied using micromanipulators (Signatone) with tungsten probes (ZN50R $\mathrm{DC} / \mathrm{RF}$ ) and the measurement was controlled by homemade LabView program via a source meter (Keithley 6430, Keithley Instruments). Silicon was wired bonded with $\mathrm{Al}$ wires. During measurements, the silicon was grounded, and the Au electrodes were biased. The applied voltage ranged from -1.0 to $+1.0 \mathrm{~V}$ with steps of $10 \mathrm{mV}$. Optical measurements were performed with an inverted optical microscope (Nikon Eclipse Ti-E) coupled with an Andor spectrometer (Shamrock 122 303i) and an electron multiplying CCD (EMCCD, iXon Ultra 897). Both the EMCCD images and spectra were collected with $2 \mathrm{~min}$ integration time following similar procedures as described in ref 7 and 8 .

\section{ASSOCIATED CONTENT}

\section{S1 Supporting Information}

The Supporting Information is available free of charge at https://pubs.acs.org/doi/10.1021/acsphotonics.0c01913.

Details of the device fabrication (Section 1), AFM measurements (Section 2), ellipsometry measurements (Section 3), and electrical measurements (Section 4). Details of the optical measurement setup is described in Section 5. Spectral fitting and cutoff analysis of the emission spectra are discussed in Section 6. A detailed note on the local density of the optical states calculations and FEM mode analysis are included in Section 7 (PDF)

\section{AUTHOR INFORMATION}

\section{Corresponding Author}

Christian A. Nijhuis - Department of Chemistry, National University of Singapore Graduate School for Integrative Sciences and Engineering, and Centre for Advanced $2 \mathrm{D}$ Materials and Graphene Research Centre, National University of Singapore, Singapore 117543, Singapore; Department of Molecules and Materials, MESA+ Institute for Nanotechnology, Faculty of Science and Technology, University of Twente, 7500 AE Enschede, The Netherlands; ○ orcid.org/0000-0003-3435-4600; Email: chmnca@ nus.edu.sg

\section{Authors}

Fangwei Wang - Department of Chemistry, National University of Singapore, Singapore 117543, Singapore

Yan Liu - Department of Electrical and Computer Engineering, 4 Engineering Drive 3, National University of Singapore, Singapore 117576, Singapore

Thorin Jake Duffin - Department of Chemistry and National University of Singapore Graduate School for Integrative Sciences and Engineering, National University of Singapore, Singapore 117543, Singapore

Vijith Kalathingal - Department of Chemistry and Centre for Advanced 2D Materials and Graphene Research Centre, National University of Singapore, Singapore 117543, Singapore

Siping Gao - Department of Electrical and Computer Engineering, 4 Engineering Drive 3, National University of Singapore, Singapore 117576, Singapore; 이이이.org/00000002-9355-8518

Wenrui Hu - Department of Electrical and Computer Engineering, 4 Engineering Drive 3, National University of Singapore, Singapore 117576, Singapore

Yongxin Guo - Department of Electrical and Computer Engineering, 4 Engineering Drive 3, National University of Singapore, Singapore 117576, Singapore

Soo-Jin Chua - Department of Electrical and Computer Engineering, 4 Engineering Drive 3, National University of Singapore, Singapore 117576, Singapore; LEES Program, Singapore-MIT Alliance for Research and Technology (SMART), Singapore 138602, Singapore

Complete contact information is available at:

https://pubs.acs.org/10.1021/acsphotonics.0c01913

\section{Author Contributions}

${ }^{\#}$ These authors contributed equally to this work.

Notes

The authors declare no competing financial interest.

\section{ACKNOWLEDGMENTS}

The authors acknowledge the National Research Foundation (NRF) for supporting this research under the Prime Minister's Office, Singapore, under its Medium Sized Centre Programme and the Competitive Research Programme (CRP; NRFCRP17-2017-08). This work is also supported by China Scholarship Council (CSC). The authors also thank Centre for Advanced 2D Materials (CA2DM) for the provided facilities. 
We thank Drs. Lu Ding, Dongyang Wan, and Yu Cao who have kindly provided the constructive suggestions in devices fabrication and improvement. All relevant data are available from the authors on request.

\section{REFERENCES}

(1) Harter, T.; Muehlbrandt, S.; Ummethala, S.; Schmid, A.; Nellen, S.; Hahn, L.; Freude, W.; Koos, C. Silicon-Plasmonic Integrated Circuits for Terahertz Signal Generation and Coherent Detection. Nat. Photonics 2018, 12 (10), 625-633.

(2) Ozbay, E. Plasmonics: Merging Photonics and Electronics at Nanoscale Dimensions. Science 2006, 311 (5758), 189-193.

(3) Huang, K. C. Y.; Seo, M.-K.; Sarmiento, T.; Huo, Y.; Harris, J. S.; Brongersma, M. L. Electrically Driven Subwavelength Optical Nanocircuits. Nat. Photonics 2014, 8 (3), 244-249.

(4) Szentirmay, Z. Surface Plasmon Assisted Electron-Photon Interaction in Metal-Oxide-Metal Layered Structures. Prog. Quantum Electron. 1991, 15 (3), 175-230.

(5) Lambe, J.; McCarthy, S. L. Light Emission from Inelastic Electron Tunneling. Phys. Rev. Lett. 1976, 37 (14), 923-925.

(6) Duffin, T. J.; Kalathingal, V.; Radulescu, A.; Li, C.; Pennycook, S. J.; Nijhuis, C. A. Cavity Plasmonics in Tunnel Junctions: Outcoupling and the Role of Surface Roughness. Phys. Rev. Appl. 2020, 14, 044021.

(7) Du, W.; Wang, T.; Chu, H.-S.; Nijhuis, C. A. Highly Efficient On-Chip Direct Electronic-Plasmonic Transducers. Nat. Photonics 2017, 11 (10), 623-627.

(8) Makarenko, K. S.; Hoang, T. X.; Duffin, T. J.; Radulescu, A.; Kalathingal, V.; Lezec, H. J.; Chu, H.; Nijhuis, C. A. Efficient Surface Plasmon Polariton Excitation and Control over Outcoupling Mechanisms in Metal-Insulator-Metal Tunneling Junctions. Adv. Sci. 2020, 7, 1900291.

(9) Zhang, C.; Hugonin, J.-P.; Greffet, J.-J.; Sauvan, C. Surface Plasmon Polaritons Emission with Nanopatch Antennas: Enhancement by Means of Mode Hybridization. ACS Photonics 2019, 6, 2788.

(10) Parzefall, M.; Bharadwaj, P.; Jain, A.; Taniguchi, T.; Watanabe, K.; Novotny, L. Antenna-Coupled Photon Emission from Hexagonal Boron Nitride Tunnel Junctions. Nat. Nanotechnol. 2015, 10 (12), $1058-1063$.

(11) Maier, S. Plasmonics: Fundamentals and Applications, 1st ed.; Springer: Bath, 2007, DOI: 10.1007/0-387-37825-1.

(12) Abeles, F. Optical Properties of Solids; North-Holland Pub. Co., 1972

(13) Lin, C. C. C.; Chang, P. H.; Su, Y.; Helmy, A. S. Monolithic Plasmonic Waveguide Architecture for Passive and Active Optical Circuits. Nano Lett. 2020, 20 (5), 2950-2957.

(14) Lafone, L.; Sidiropoulos, T. P. H.; Oulton, R. F. Silicon-Based Metal-Loaded Plasmonic Waveguides for Low-Loss Nanofocusing. Opt. Lett. 2014, 39 (15), 4356-4359.

(15) Oulton, R. F.; Sorger, V. J.; Genov, D. A.; Pile, D. F. P.; Zhang, X. A Hybrid Plasmonic Waveguide for Subwavelength Confinement and Long-Range Propagation. Nat. Photonics 2008, 2 (8), 496-500.

(16) Chen, M.-J.; Liang, E.-Z.; Chang, S.-W.; Lin, C.-F. Model for Band-Edge Electroluminescence from Metal-Oxide-Semiconductor Silicon Tunneling Diodes. J. Appl. Phys. 2001, 90 (2), 789-793.

(17) Chang, S.; Chen, K.; Shie, C.; Liu, C.; Chen, M.-J.; Lin, C.-F. The Band-Edge Light Emission from the Metal-Oxide-Silicon Tunneling Diode on (110) Substrates. Solid-State Electron. 2002, 46 (8), 1113-1116.

(18) Grekhov, I. V.; Shulekin, A. F.; Vexler, M. I. Visible Hot Electron Electroluminescence from $\mathrm{Si}$ in Tunnel MIS Junction. Proceedings of the 1997 21st International Conference on Microelectronics; IEEE, 1997; Vol. 1, pp 165-168, DOI: DOI: 10.1109/ ICMEL.1997.625206

(19) Liu, C. W.; Lee, M. H.; Lin, C. F.; Lin, I. C.; Liu, W. T.; Lin, H. H. Light Emission and Detection by Metal Oxide Silicon Tunneling Diodes. IEEE Int. Electron Devices Meet.; IEEE, 1999; pp 749-752, DOI: $10.1109 /$ IEDM.1999.824259.
(20) Lin, C. F.; Liu, C. W.; Chen, M. J.; Lee, M. H.; Lin, I. C. Electroluminescence at Si Band Gap Energy Based on Metal-OxideSilicon Structures. J. Appl. Phys. 2000, 87 (12), 8793-8795.

(21) Liu, C. W.; Lee, M. H.; Chen, M.-J.; Lin, I. C.; Lin, C.-F. Room-Temperature Electroluminescence from Electron-Hole Plasmas in the Metal-Oxide-Silicon Tunneling Diodes. Appl. Phys. Lett. 2000, 76 (12), 1516-1518.

(22) Lin, C. F.; Su, T. W.; Chung, P. F.; Liang, E. Z.; Chen, M. J.; Liu, C. W. Enhancing Electroluminescence from Metal-Oxide-Silicon Tunneling Diodes by Nano-Structures of Oxide Grown by LiquidPhase Method. Mater. Chem. Phys. 2003, 77 (2), 430-433.

(23) Liu, C. W.; Lee, M. H.; Chen, M.-J.; Lin, C.-F.; Chern, M. Y. Roughness-Enhanced Electroluminescence from Metal Oxide Silicon Tunneling Diodes. IEEE Electron Device Lett. 2000, 21 (12), 601603.

(24) Ran, G. Z.; Fu, J. S.; Qin, W. C.; Zhang, B. R.; Qiao, Y. P.; Qin, G. G. Blue to Red Electroluminescence from Au/Native Silicon Oxide/p-Si Structure Subjected to Rapid Thermal Annealing. Thin Solid Films 2001, 388 (1-2), 213-216.

(25) Seidel, J.; Göhler, T.; Grafström, S.; Eng, L. M. Near-Field Optical Characterization of Surface-Plasmon-Mediated Light Emission from Electrically Biased Metal-Insulator-Semiconductor Tunnel Junctions. Appl. Phys. Lett. 2008, 92 (10), 103123.

(26) Wang, M.; Yu, J.; Sun, C. Light Emission Characteristics and Negative Resistance Phenomenon of Si-Based Metal/Insulator/ Semiconductor Tunnel Junction. Appl. Surf. Sci. 2000, 161 (1), 9-13.

(27) Watanabe, J.; Uehara, Y.; Murota, J.; Ushioda, S. Light Emission from Si-Metal-Oxide-Semiconductor Tunnel Junctions. Jpn. J. Appl. Phys. 1993, 32 (Part 1, No. 1A), 99-104.

(28) Asli, N.; Vexler, M. I.; Shulekin, A. F.; Yoder, P. D.; Grekhov, I. V.; Seegebrecht, P. Threshold Energies in the Light Emission Characteristics of Silicon MOS Tunnel Diodes. Microelectron. Reliab. 2001, 41 (7), 1071-1076.

(29) Illarionov, Y. Y.; Vexler, M. I.; Fedorov, V. V.; Suturin, S. M.; Sokolov, N. S. Electrical and Optical Characterization of $\mathrm{Au} / \mathrm{CaF}_{2} / \mathrm{p}-$ Si(111) Tunnel-Injection Diodes. J. Appl. Phys. 2014, 115 (22), 223706.

(30) Yoder, P. D.; Vexler, M. I.; Shulekin, A. F.; Asli, N.; Gastev, S. V.; Grekhov, I. V.; Seegebrecht, P.; Tyaginov, S. E.; Zimmermann, H. Luminescence Spectra of an $\mathrm{Al} / \mathrm{Si} \mathrm{O} 2 / \mathrm{p}-\mathrm{Si}$ Tunnel Metal-OxideSemiconductor Structure. J. Appl. Phys. 2005, 98 (8), No. 083511.

(31) Matsumura, K.; Yamada, R.; Arima, K.; Uchikoshi, J.; Morita, M. Electroluminescence in Metal-Oxide-Semiconductor Tunneling Diodes with Ultra Thin Silicon. ECS Trans.; IOP Publishing Ltd., 2009; Vol. 25, pp 3-8, DOI: 10.1149/1.3236403.

(32) Mihaychuk, J. G.; Denhoff, M. W.; McAlister, S. P.; McKinnon, W. R.; Ma, P.; Lapointe, J.; Chin, A. Light Emission in Silicon Tunnel Diodes. Photonics North 2004: Optical Components and Devices, Proc. SPIE; SPIE, 2004; Vol. 5577, DOI: 10.1117/12.567082.

(33) Göktas, H.; Gökhan, F. S.; Sorger, V. J. Electrical-Driven Plasmon Source of Silicon Based on Quantum Tunneling. ACS Photonics 2018, 5 (12), 4928-4936.

(34) Chen, T. C.; Lai, W. Z.; Liang, C. Y.; Chen, M. J.; Lee, L. S.; Liu, C. W. Light Emission from $\mathrm{Al} / \mathrm{HfO}_{2} /$ Silicon Diodes. J. Appl. Phys. 2004, 95 (11), 6486-6488.

(35) Morita, M.; Tsuchida, A.; Matsumura, K.; Yamada, R.; Oshikane, Y.; Kawai, K.; Uchikoshi, J.; Arima, K. (Invited) Electroluminescence in Metal-Oxide-Semiconductor Tunnel Diodes with Nanometer-Thick Silicon. In ECS Trans.; IOP Publishing Ltd., 2012; Vol. 45, pp 229-234, DOI: 10.1149/1.3700431.

(36) Morita, M.; Matsumura, K.; Yamada, R.; Uchikoshi, J.; Arima, $\mathrm{K}$. (Invited) Electroluminescence in Metal-Oxide-Semiconductor Tunnel Diodes with a Silicon Nanolayer. ECS Trans. 2010, 28 (3), 279-284.

(37) Asli, N.; Vexler, M. I.; Shulekin, A. F.; Seegebrecht, P. HotElectron-Induced Luminescence of Metal-Oxide-Semiconductor Tunnel Devices. Semicond. Sci. Technol. 2003, 18 (2), 147.

(38) Versari, R.; Pieracci, A.; Manfredi, M.; Soncini, G.; Bellutti, P.; Ricco, B. Light Emission from MOS Tunnel Diodes. In IEEE Int. 
Electron Devices Meet.; IEEE, 1999; pp 745-748, DOI: 10.1109/ iedm.1999.824258.

(39) Liu, C. W.; Lee, M. H.; Chen, M.-J.; Lin, I. C.; Lin, C.-F. Room-Temperature Electroluminescence from Electron-Hole Plasmas in the Metal-Oxide-Silicon Tunneling Diodes. Appl. Phys. Lett. 2000, 76, 1516.

(40) Cartier, E.; Tsang, J. C.; Fischetti, M. V.; Buchanan, D. A. Light Emission during Direct and Fowler-Nordheim Tunneling in Ultra Thin MOS Tunnel Junctions. Microelectron. Eng. 1997, 36 (1-4), 103-106.

(41) Wang, M.; Yu, J.; Sun, C. Light Emission Characteristics and Negative Resistance Phenomenon of Si-Based Metal/Insulator/ Semiconductor Tunnel Junction. Appl. Surf. Sci. 2000, 161 (1-2), $9-13$.

(42) Uehara, Y.; Watanabe, J.; Fujikawa, S.; Ushioda, S. LightEmission Mechanism of Si-MOS Tunnel Junctions. Phys. Rev. B: Condens. Matter Mater. Phys. 1995, 51 (4), 2229-2238.

(43) DiMaria, D. J.; Cartier, E.; Arnold, D. Impact Ionization, Trap Creation, Degradation, and Breakdown in Silicon Dioxide Films on Silicon. J. Appl. Phys. 1993, 73 (7), 3367-3384.

(44) Sire, C.; Blonkowski, S.; Gordon, M. J.; Baron, T. Statistics of Electrical Breakdown Field in $\mathrm{HfO}_{2}$ and $\mathrm{SiO}_{2}$ Films from Millimeter to Nanometer Length Scales. Appl. Phys. Lett. 2007, 91, 242905.

(45) Dawson, P.; Walmsley, D. G.; Quinn, H. A.; Ferguson, A. J. L. Observation and Explanation of Light-Emission Spectra from Statistically Rough $\mathrm{Cu}, \mathrm{Ag}$, and $\mathrm{Au}$ Tunnel Junctions. Phys. Rev. B: Condens. Matter Mater. Phys. 1984, 30 (6), 3164-3178.

(46) Sze, S. M.; Ng, K. K. Physics of Semiconductor Devices, 3rd ed.; Wiley: Hoboken, NJ, 2007, DOI: 10.1002/0470068329.

(47) Davis, L. C. Theory of Surface-Plasmon Excitation in MetalInsulator-Metal Tunnel Junctions. Phys. Rev. B 1977, 16 (6), 24822490

(48) Buret, M.; Uskov, A. V.; Dellinger, J.; Cazier, N.; Mennemanteuil, M.-M.; Berthelot, J.; Smetanin, I. V.; Protsenko, I. E.; Colas-des-Francs, G.; Bouhelier, A. Spontaneous Hot-Electron Light Emission from Electron-Fed Optical Antennas. Nano Lett. 2015, 15 (9), 5811-5818.

(49) Downes, A.; Dumas, P.; Welland, M. E. Measurement of High Electron Temperatures in Single Atom Metal Point Contacts by Light Emission. Appl. Phys. Lett. 2002, 81 (7), 1252-1254.

(50) Malinowski, T.; Klein, H. R.; Iazykov, M.; Dumas, P. Infrared Light Emission from Nano Hot Electron Gas Created in Atomic Point Contacts. Europhys. Lett. 2016, 114 (5), 57002.

(51) Zhu, Y.; Cui, L.; Natelson, D. Hot-Carrier Enhanced Light Emission: The Origin of above-Threshold Photons from Electrically Driven Plasmonic Tunnel Junctions. J. Appl. Phys. 2020, 128 (23), 233105.

(52) Parzefall, M.; Szabó, Á.; Taniguchi, T.; Watanabe, K.; Luisier, M.; Novotny, L. Light from van Der Waals Quantum Tunneling Devices. Nat. Commun. 2019, 10 (1), 292.

(53) Gurunarayanan, S. P.; Verellen, N.; Zharinov, V. S.; James Shirley, F.; Moshchalkov, V. V.; Heyns, M.; Van De Vondel, J.; Radu, I. P.; Van Dorpe, P. Electrically Driven Unidirectional Optical Nanoantennas. Nano Lett. 2017, 17 (12), 7433-7439.

(54) Dionne, J. A.; Diest, K.; Sweatlock, L. A.; Atwater, H. A. PlasMOStor: A Metal-Oxide-Si Field Effect Plasmonic Modulator. Nano Lett. 2009, 9 (2), 897-902.

(55) Thompson, T. D.; Allen, J. W. Electroluminescence from Minority Carrier Injection Produced by Deep-Level ImpactIonisation. J. Cryst. Growth 1990, 101 (1-4), 981-984.

(56) Cheung, S. K.; Cheung, N. W. Extraction of Schottky Diode Parameters from Forward Current-Voltage Characteristics. Appl. Phys. Lett. 1986, 49 (2), 85-87.

(57) Card, H. C.; Rhoderick, E. H. Studies of Tunnel MOS Diodes I. Interface Effects in Silicon Schottky Diodes. J. Phys. D: Appl. Phys. 1971, 4 (10), 319.

(58) Ford, G. W. W.; Weber, W. H. H. Electromagnetic Interactions of Molecules with Metal Surfaces; North-Holland, 1984; Vol. 113, pp 195-287, DOI: 10.1016/0370-1573(84)90098-X.
(59) Aspnes, D. E.; Studna, A. A.; Kinsbron, E. Dielectric Properties of Heavily Doped Crystalline and Amorphous Silicon from 1.5 to 6.0 EV. Phys. Rev. B: Condens. Matter Mater. Phys. 1984, 29 (2), 768-779.

(60) Olmon, R. L.; Slovick, B.; Johnson, T. W.; Shelton, D.; Oh, S. H.; Boreman, G. D.; Raschke, M. B. Optical Dielectric Function of Gold. Phys. Rev. B: Condens. Matter Mater. Phys. 2012, 86 (23), 235147.

(61) COMSOL Inc. COMSOL Multiphysics; COMSOL AB: Stockholm, Sweden.

(62) Brinkman, W. F.; Dynes, R. C.; Rowell, J. M. Tunneling Conductance of Asymmetrical Barriers. J. Appl. Phys. 1970, 41 (5), $1915-1921$.

(63) Wu, C. Y. Interfacial Layer Theory of the Schottky Barrier Diodes. J. Appl. Phys. 1980, 51 (7), 3786-3789. 